\title{
MMS over MQTT Protocol for Industrial IoT Platform
}

\author{
[ Hea-Min Lee, Seung-Han Kim, Joong-Hyuk Cha and Dong-Seong Kim* ]
}

\begin{abstract}
In this paper, MoM(MMS over MQTT) protocol proposed for industrial IoT(Internet of Things) platform. The MMS(Manufacturing Message Specification), that is used for conventional industrial platform, can control only one device by one message. Because of this, when control many devices at same time, message publisher build message for each target devices. So, unnecessary delay is occurred while building messages and sending messages. And industrial devices, which use private IP(Internet Protocol) address, can't use MMS and it is not commensurate with industrial IoT platform. So, MQTT(MQ Telemetry Transport) is introduced for overcome these weakness. Proposed scheme build MMS packet and using MQTT for communication. For implement test bed, intel edison board is used. Test bed communicate each other and actuators are controlled by value of sensors.
\end{abstract} Time

Keywords-Industrial IoT platform; MMS; MQTT; Real-

\section{Introduction}

Industrial site changed in recent year. The Industrial devices such as sensors or actuators are construct industrial IoT through combine with local area network[1], wireless sensor network, mobile technology. These technology is designed for managing industrial environment or product line in industrial site. The majority of industrial IoT research focus on these technology[2][3]. Conventional MMS, which used for industrial platform, is provide unified message for industrial devices. Through this, application developer or factory manager can reduce their cost of developing or managing devices. Even though this advantage, conventional MMS invalid for industrial IoT platform. It use TCP/IP based communication and impossible to operate with private IP address[4]. Furthermore, MMS have to building new message for control each devices and it occurs unnecessary delay. In this paper, MoM protocol is designed and implemented for overcome disadvantage of conventional MMS. Proposed protocol use MMS packet and broker based transmission. Through numerical equation we can confirmed MoM protocol can reduce end to end delay compare with MMS. And operation of protocol also confirmed by building test bed using intel edison board.

\section{Hea-Min Lee}

Dept of Electronic Engineering, Kumoh National Institute of Technology Gumi-City, South Korea

\section{Seung-Han Kim}

Dept of Electronic Engineering, Kumoh National Institute of Technology Gumi-City, South Korea

Joong-Hyuk Cha

Dept of Electronic Engineering, Kumoh National Institute of Technology Gumi-City, South Korea

Dong-Seong Kim

Dept of Electronic Engineering, Kumoh National Institute of Technology Gumi-City, South Koreaa

\section{MoM Protocol Design for Industrial IoT Platform}

\section{A. Disadvantage of Conventional MMS for apply to Industrial IoT Platform}

The MMS can improve choices and flexibility of industrial devices through unify message standard between kind of industrial devices. In addition, using single protocol, industrial systems development and maintenance costs are reduced[5].

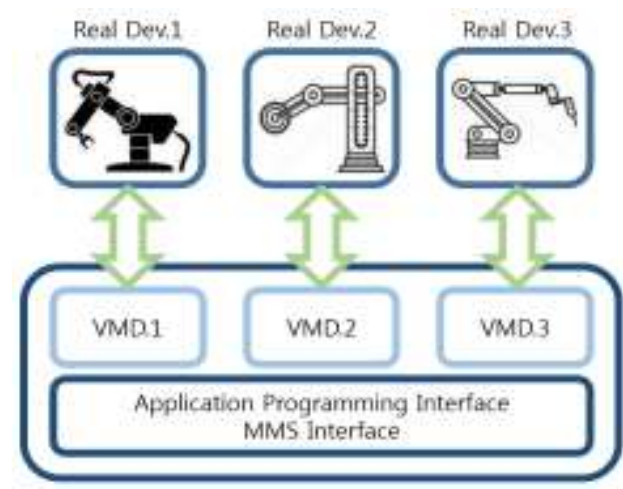

Figure 1. MMS Function Structure

However, the MMS have two disadvantages to apply in industrial IoT platform. At first, only one device can be controlled as one message. For controlling another device, modifying the message address and re-issued message again. As a result, such a message must be repeatedly transmitted in order to control multiple devices with the same instruction, this increases the end-to-end delay. Secondly, a fixed IP environment, can be used only and does not support the communication of the device using a private IP address. Due to these characteristics the MMS is not suitable for many-to-one and many-to-many communication or industrial IoT platform with an industrial local area networks and wireless sensor networks that use private IP.

\section{B. MoM Protocol Design for Industrial IoT Platform}

In order to apply the MMS to industrial IoT platform, it should complement the two drawbacks mentioned above. In this paper, introduce MQTT for overcome disadvantage of MMS.

MQTT is a protocol that exchanging publication and subscription messages using center broker[6]. MQTT is constructed by publisher(PUB), subscriber(SUB), broker. In the case of PUB, In the case of PUB, it publish message to 


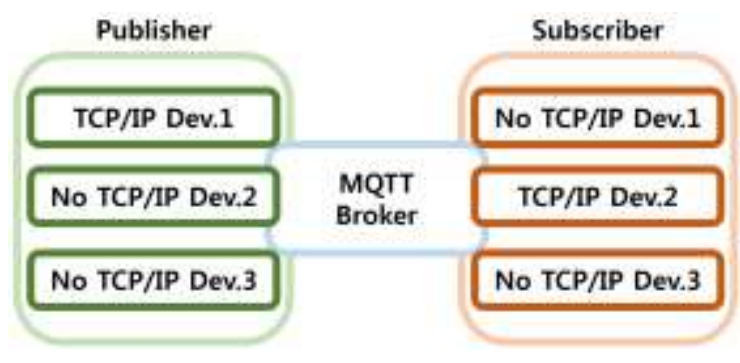

Figure 2. Basic MQTT structure

broker which have a set topic and SUB is subscribe a message with the same set Topic. And broker manage these process. MQTT-based network can be easily parallelized because broker acts as an event-driven method. Through this, industrial device, which use MMS, can reduce unnecessary delay for transfer command to large number of devices. Also the broker of MQTT may MMS can operate on various network environment(e.g. wireless sensor network, industrial local area network, etc.).

The proposed protocol is operated in such a manner as to exchange MMS protocol data unit(PDU) using the MQTT protocol. MoM protocol is divided as PUB Layer protocol which use ASN.1 encoder and SUB Layer protocol which use ASN.1 decoder because MoM protocol use ASN.1 standard. Operation of proposed protocol is based on MMS. So, service of MMS(e.g. program operation management, event/journal management, user communication service, etc.) can use in proposed protocol.

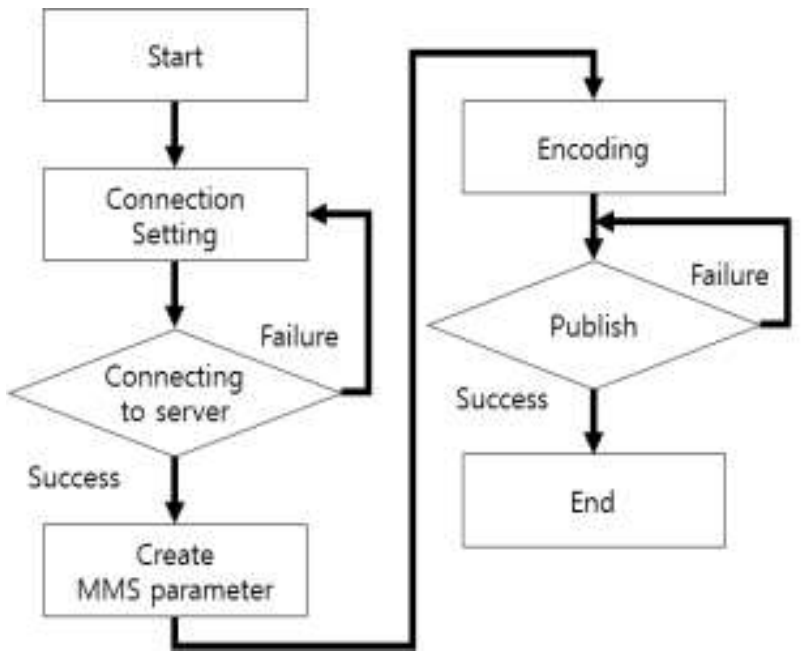

Figure 3. PUB Layer Protocol Flowchart

Operation of PUB Layer protocol as follows. Firstly, initialize processing for connect to MQTT server is proceeds through connection option setting function. At this stage settings Alive Interval, Clean Session, QoS level, etc. And callback function that is called when the connection is successful or fails is set. If PUB is connected with server, set physical/logical state and PDU type of virtual machine device(VMD). This stage is needed for provide MMS service to VMD. If PUB is industrial device, the state of VMD or value of event is included to MMS PDU. In contrast, PUB is control system, the message that is order to VMD is included. After setting issue information to MMS PDU, encode the message using MMS encoder. After that message is transferred through MQTT and process is closed.

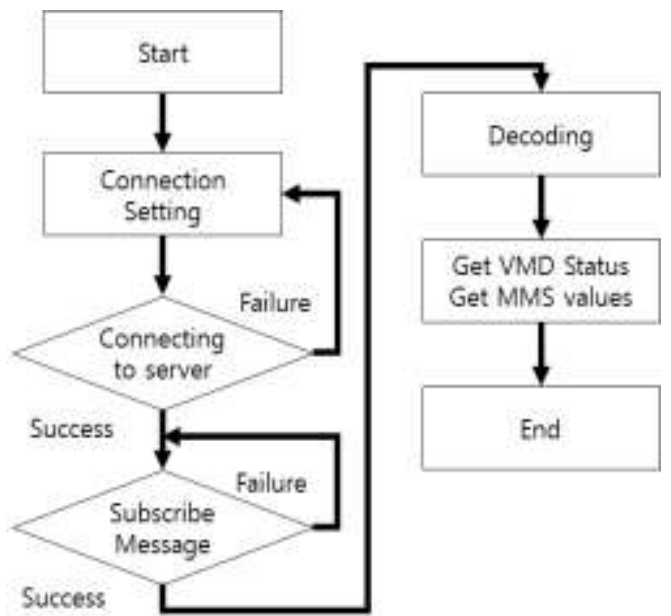

Figure 4. SUB Layer Protocol Flowchart

The case of SUB Layer protocol as follows. Firstly, initialize processing is same with PUB Layer protocol. After that when SUB is connected with server, SUB try to subscribe message which have same topic with SUB. If message is subscribed, ASN.1 decoder is called and decode message. Through this stage SUB can get data from MMS PDU and control devices. Finally get issue information from PDU and close the process.

\section{Experimental Results}

In order to check operation of protocol, in this section, MoM protocol is implemented by intel edison board. Intel edison board provide wifi connection that can easily connect to MQTT broker. Whole structure of test bed is presented in Figure 5. In publisher, temperature sensor and switch are installed. And subscriber, servomotor and LED are installed.

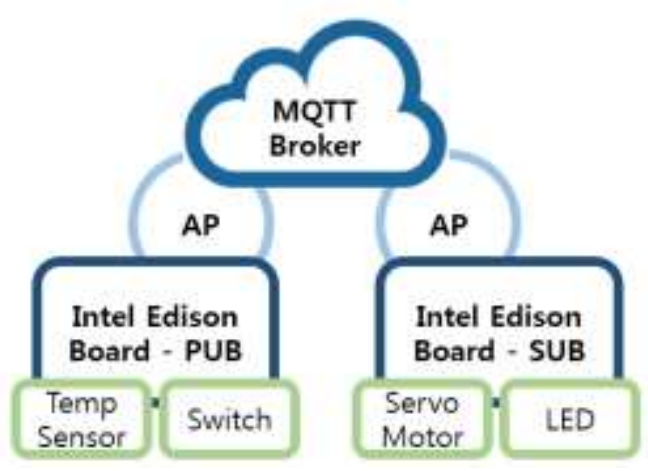

Figure 5. Test Bed Structure Diagram

\section{A. Pub/Sub Firmware Implementation and Upload}

Refer to designed protocols for implement firmware. So, flowchart of firmware are almost same with flowchart of protocol. The sequence of operations of the publisher firmware are: 1) Connection Setting(WiFi, MQTT broker address, etc.), 2) Try to connect to MQTT broker. 3) Get value from temperature sensor and switch. 4) Build MMS PDU and encoding message. 5) Publishing message to MQTT broker. 
And the sequence of operations of the subscriber firmware are: 1) Connection Setting(WiFi, MQTT broker address, etc.), 2) Try to connect to MQTT broker. 3) Subscribe message from broker. 4) Decoding message and get data from MMS PDU.

Source coding and uploading was proceeded through the Arduino sketch.

\section{B. Check Operation of Protocol}

Operation of protocol is confirmed using arduino skech. Left window of Figure 6 is publisher which publishing message and right window is subscriber which subscribing message.

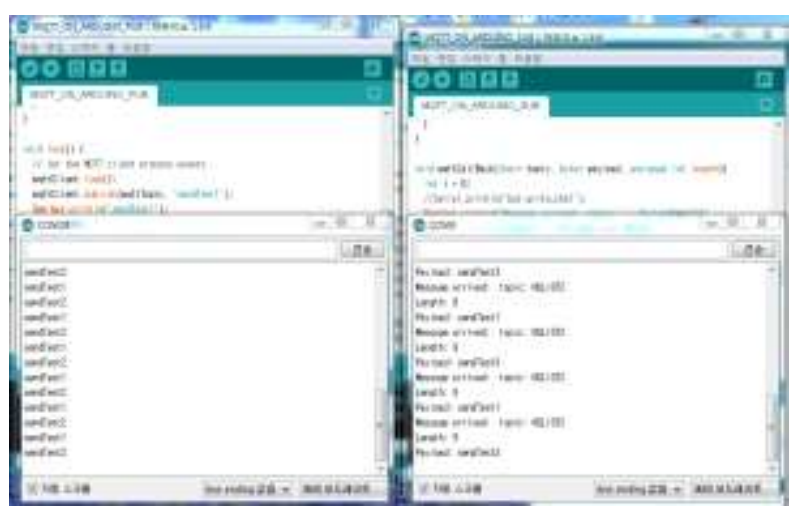

Figure 6. Operation of Protocol Check via Arduino Sketch

\section{Conclusion}

In this paper MoM protocols are designed and implemented. To verify the possibility as industrial IoT platform and its operation, proposed protocol was implemented using Inter Edison Board. Advantages of proposed protocol are as follows. Firstly, using MQTT, the efficient of many-to-one and many-to-many communication are improved. By introducing MQTT was able to provide the MMS service in the private IP environment.

After design protocol, according protocol design, test bed is constructed. Test bed can publish MMS message through MQTT broker and subscribe from MQTT broker. Through this, operation of implemented firmware is confirmed.

\section{Acknowledgment}

This research was financially supported by the Ministry of Education, Science Technology (MEST) and National Research Foundation of Korea(NRF) through the Human Resource Training Project for Regional Innovation (NO. NRF-2015H1C1A1035971).

\section{References}

[1] Zhengguo Sheng, etc., "Recent Advances in Industrial WSN toword Efficient Management in IoT," IEEE Access, Vol. 3, pp. 622-637, May 2015.

[2] Qingping Chi, Hairong Yan, Chuan Zhang, Zhibo Pang, and Li Da $\mathrm{Xu}$, "A Reconfigurable Smart Sensor Interface for Industrial WSN in IoT Environment," IEEE Transactions on Industrial Informatics, Vol. 10, No. 2, pp. 1417-1425, Feb. 2014. I. S. Jacobs and C. P. Bean,
"Fine particles, thin films and exchange anisotropy," in Magnetism, vol. III, G. T. Rado and H. Suhl, Eds. New York: Academic, 1963, pp. 271-350.

[3] Q. Zhu, R. Wang, Q. Chen, Y. Liu, and W. Qin, "IoT gateway: Bridging wireless sensor networks into internet of things," in Proc. IEEE/IFIP 8th International Conference Embedded Ubiquitous Computing (EUC), Hong Kong, China, pp. 347-352, 11-13 Dec. 2010. R. Nicole, "Title of paper with only first word capitalized," J. Name Stand. Abbrev., in press.

[4] Ungurean, Gaitan, N.-C., Gaitan, V.G. "An IoT Architecture for Things from Industrial Environment," International Conference on Communications(COMM), pp. 1-4, Bucharest, 29-31 May 2014. M. Young, The Technical Writer's Handbook. Mill Valley, CA: University Science, 1989.

[5] Uk-Hyun Gwon, Dong-Seong Kim, "To practice in a systematic analysis of the understanding and application of MMS," OHM, pp. 1583, 2008.

[6] Seung-Hyun Sim, Hak-Bum Kim, "IoT and MQTT Technology," KIISC, Vol. 24, No. 6, pp. 37-47, Dec. 2014.

About Author (s):

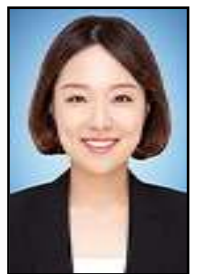

She was a Master student at IT Convergence, School of Electronics Engineering, Kumoh National Institute of Technology, South Korea. She currently research work in ETRI. Her research interests are wireless sensor networks and

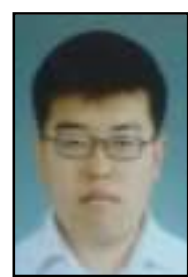

$\mathrm{He}$ is a Master student at Electronics Engineering, School of Electronics Engineering, Kumoh National Institute of Technology, South Korea. His research interests are wireless sensor networks and embedded systems.

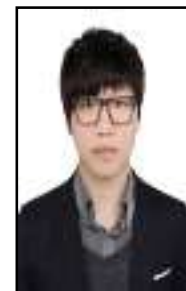

He is a Master student at IT Convergence Department, School of Electronics Engineering, Kumoh National Institute of Technology, South Korea. His research interests are wireless sensor networks and embedded systems.

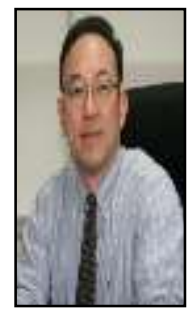

Dong-Seong Kim received his Ph.D degree in Electrical and Computer Engineering from the Seoul National University, Seoul, Korea, in 2003. From 1994 to 2003, he worked as a full-time researcher in ERC-ACI at Seoul National University, Seoul, Korea. From March 2003 to February 2005, he worked as a postdoctoral researcher at the Wireless Network Laboratory in the School of Electrical and Computer Engineering at Cornell University, NY. He is currently a director of ICT Convergence Research Center supported by Korean government at Kumoh National Institute of Technology. His current main research interests are industrial wireless control network, networked embedded system and Fieldbus. 